\title{
Investigation of Assembly Forces During Creation of Head-Neck Taper Junction Following Total Hip Arthroplasty: A Biomechanical Study
}

Toni Wendler ( $\boldsymbol{\nabla}$ toni.wendler@medizin.uni-leipzig.de )

Universitat Leipzig Medizinische Fakultat https://orcid.org/0000-0001-7890-178X

Torsten Prietzel

Zeisigwaldkliniken Bethanien Chemnitz

Robert Möbius

Universitat Leipzig Medizinische Fakultat

Jean-Pierre Fischer

Universitat Leipzig Medizinische Fakultat

Andreas Roth

Universitat Leipzig Medizinische Fakultat

Dirk Zajonz

Zeisigwaldkliniken Bethanien Chemnitz

Research article

Keywords: Assembly force, Head-neck taper junction, Soft tissue damping, Total hip arthroplasty, biomechanical study

Posted Date: August 26th, 2020

DOI: https://doi.org/10.21203/rs.3.rs-62417/v1

License: (c) (i) This work is licensed under a Creative Commons Attribution 4.0 International License. Read Full License 


\section{Abstract}

\section{Background}

All current total hip arthroplasty (THA) systems are modular in design. Only during the operation femoral head and stem get connected by a Morse taper junction. The junction is realized by hammer blows from the surgeon. Decisive for the junction strength is the maximum force acting once in direction of the neck axis, which depends both on the applied impulse and the damping of human soft tissues. This leads to large differences in assembly forces between the surgeons. The investigation of assembly forces of different surgeons under influence of soft tissue damping is subject of this study.

\section{Methods}

First, a measuring system, consisting of a prosthesis and a hammer, was developed. Both components are equipped with a piezoelectric force sensor. Initially, in situ experiments on human cadavers were carried out using this system in order to determine the actual assembly forces and to characterize the damping properties of human soft tissue. In addition to these experiments, an in vitro model in form of an artificial femur (Sawbones Europe AB, Malmo, Sweden) with implanted measuring stem embedded in gelatine was developed. The gelatine mixture was chosen in such a way that damping properties of the model correspond to those in situ. A study with 31 surgeons was carried out on the in vitro model mentioned above, in which the assembly forces were determined.

\section{Results}

A model has been developed, that represents the physiological damping behaviour of human soft tissue. The assembly forces measured on in vitro model were on average $2037.2 \mathrm{~N} \pm 724.9 \mathrm{~N}$ and ranged from $822.5 \mathrm{~N}$ to $3835.2 \mathrm{~N}$. The comparison of the surgeons showed no significant differences regarding sex $(p=0.09)$, work experience $(p=0.71)$ and number of THAs per year $(p=0.69)$.

\section{Conclusions}

All measured assembly forces were below $4 \mathrm{kN}$, which is recommended in the literature. This could lead to increased corrosion following fretting in the head-neck interface. In addition, there was a very wide range of assembly forces among the surgeons, although other influencing factors such as different implant sizes or materials were not taken into account. To ensure optimal assembly force, the impaction should be standardized, e.g. by an appropriate surgical instrument.

\section{Background}

The replacement of a degenerated joint with an artificial joint is an extremely successful medical procedure, during which most patients regain their mobility within a short time and experience significant pain relief. The use of an artificial joint is therefore one of the standard surgeries in modern medicine 
today [1]. Several hundred thousand hip endoprostheses are implanted worldwide every year [2-5] with sharp increase in demand $[5,6]$.

Current THA systems are modular in design to provide the patient with the ideal clinical treatment. For example, the material of the bearing partners can be varied or the individual anatomy of the patient can be taken into account. In hip endoprosthetics almost all implant manufacturers use the principle of a Morse taper junction.

However, in the recent past there have been increasing reports of problems with taper junctions, which are usually accompanied by corrosion and abrasion. Especially metal-on-metal bearings are affected [7-9] but also ceramic bearings [10]. Such problems occur in primary [11] but also in revision THA [12].

In scientific literature there are some references to the causes of failure. Explant studies have shown that impurities in the head-neck interface reduce its strength and increase the risk of corrosion [13]. Furthermore, the material and the assembly forces seem to play an important role $[14,15]$.

During implantation, the head-neck taper junction is joined by one or more hammer blows. The hammer has an impulse, which is partially or completely transmitted to the impactor tool and the femoral head upon impact. The change in momentum of the hammer corresponds to the integral of the measured force over time. At the moment of impact the hammer is abruptly decelerated (negatively accelerated). According to Euler's definition of force, the force is the product of mass and acceleration. Despite the relatively low mass of the hammer head, the high negative acceleration results in high forces of several kilonewtons for a short time. Decisive for the junction strength is the maximum force acting once in direction of the neck axis [14].

However, it must be assumed that such a hammer blow is performed very individually by the surgeon and is therefore not reproducible. This is illustrated by Nasutt et al. [16] who compared the assembly forces resulting from hammer blows of 39 surgeons. It was shown that the force varies between $0.27 \mathrm{kN}$ and $7.85 \mathrm{kN}$ [16]. However, these were only trials on a test rig, so it must be assumed that the absolute values in surgery are different due to soft tissue damping. Nevertheless, the study shows that the assembly forces can vary greatly between surgeons and that this influences the strength of a Morse taper junction $[14,15]$. The aim of this study was to investigate the assembly forces of different surgeons under in situ conditions.

\section{Methods}

\section{Development of a suitable measurement system}

First, it was necessary to develop a measuring system capable of precisely measuring the highly dynamic forces occurring when joining head and the neck of the stem. For this purpose piezoelectric sensors from PCB Piezotronics (Depew, NY, USA) were used. Piezoelectric sensors can measure high frequency force signals and are available in small sizes. The housings of the sensors used are still hermetically sealed so 
they can be used in situ. A sensor (PCB 208C05) was integrated into the neck of a CBC Evolution stem from Mathys (Bettlach, Switzerland), as shown in Fig. 1a). The sensor was mounted distally and proximally force-locked via thread bolts. Thus, it directly measured the force acting on the taper. Geometric parameters of the stem, like CCD angle or offset, were not changed by inserting the sensor. The CBC Evolution is a standard non-cemented Spotorno stem and is laterally symmetrical, i.e. it can be implanted on both left and right side. This allowed one stem to be used for both sides, which significantly reduced material costs.

Since the forces occurring during impaction largely depend on damping of the entire system, especially of human soft tissues, the measuring system should be able to determine the damping behaviour. For this reason, an acceleration sensor was attached to the top side of the taper. It should determine the impulse response of the system from which the damping behaviour can be calculated. The sensor was a 3-axis acceleration sensor (PCB 356A03/NC).

Another component of the measuring system was an impulse hammer (PCB TLD086D05), which measured the forces on the impactor during the impaction. The hammer used is shown in Fig. 1b). It was equipped with an additional weight in order to obtain the same weight $(416 \mathrm{~g})$ as a Bergmann mallet.

Both sensors were connected to a signal conditioner (PCB 482C15), which supplied the sensors and amplified the measuring signals. A Data Acquisition (DAQ) module digitized the analogue signals and transmitted them to a Personal Computer (PC), where the data was recorded by software specially developed in LabView (NI, Austin, USA).

Figure 1. a) Stem with integrated piezoelectric force sensor; b) Impulse hammer

\section{Experimental impactions on human cadavers}

First the developed measuring system was used to carry out a series of experiments on cadavers. For this purpose the measuring prosthesis was implanted in chemically untreated fresh human cadavers (Fig. 2 left). All implantations were performed by an experienced senior physician via a lateral transgluteal approach. The ceramic head, a $36 \mathrm{~mm}$ ceramys head size L (Mathys AG, Bettlach, Switzerland) was placed on the neck of the implanted stem and was then impacted by a hammer blow via the attached impactor tool (CBC head impactor, Mathys AG, Bettlach, Switzerland). The entire setup is shown in Fig. 2 right. The resulting forces and accelerations were recorded as described previously. Furthermore, the proximal femur was examined for possible fractures following impaction.

Figure 2. left: Implanted measuring stem; right: Setup to measure in situ assembly forces

The procedure was performed on five hips from three different body donors. One cadaver had an implanted femoral nail at the left side. In case there was no previous surgical treatment of the hip, the measuring stem was implanted on both sides. The impaction was performed by three different surgeons. Surgeon I was an experienced senior physician, surgeon II a specialist physician and surgeon III an assistant physician. Surgeon I impacted three times and surgeon II and III impacted once each. The body 
donors were 71, 83 and 84 years old and weighed $54 \mathrm{~kg}, 65 \mathrm{~kg}$ and $92 \mathrm{~kg}$. Since untreated fresh human cadavers were to be examined the number of experiments was limited due to the small time window in which recruiting surgeons and carrying out the experiments were possible. In order to be independent of the restrictions associated with the use of cadavers and yet increase the number of experiments $\mathrm{N}$ the obtained data were used to develop an analogous in vitro model.

\section{Development of an in vitro model}

In order to obtain valid data using the analogous in vitro model the main focus has been the reproduction of the damping behaviour as measured on the cadavers. Furthermore, the orientation and alignment of the setup played an important role. In particular impact height or angles deviating from normal surgical procedures on the patient might falsify the measurement results. To meet these special requirements the sensor-equipped stem was first implanted into a 4th generation artificial femur (Sawbones, Vashon Island, Washington; USA) by the same experienced surgeon as in the cadaver experiments. This femur was then embedded in a block of gelatine in such a way that the alignment and orientation of bone and stem corresponded to a THA with lateral transgluteal approach (Fig. 3).

\section{Figure 3. Schematic of embedded Femur with implanted THA stem}

The gelatine block was designed to reproduce the damping behaviour of human soft tissue. It was cast in a housing made of polymethylmethacrylate (PMMA), which was rigidly screwed to a profile frame. As in the experiments on the cadavers, the sensors of the measuring system were connected to a signal conditioner and PC. The final in vitro model is shown in Fig. 4.

\section{Figure 4. Final in vitro model}

The mixing ratio of gelatine granulate and water determines the resulting stiffness of the gelatine and thus its damping. The aim was to produce the same properties as those present in situ. For the purpose of finding the optimal mixing ratio of the gelatine the in vitro model was built with mixing ratios of $1: 4$ and 1:9 (parts of gelatine:parts of water). As in the experiments on human cadavers a ceramic head was placed on the neck of the stem and then impacted by a hammer blow. For each mixing ratio 10 impactions were performed. The measured data were compared with those of the experiments on cadavers.

The first approach to quantify the damping behaviour was to use the impulse responses of the accelerometer to calculate the parameters of a simple spring mass damper system which, however, did not reflect the physiological conditions sufficiently well. It was then attempted to parameterize a threestage spring mass damper system to better reflect the measured impulse responses. It was found that such a parameterization is possible in principle but not practical, since it requires a lot of time and parameters of the measured impulse response vary greatly between the individual human cadavers. In order to still be able to compare the damping behaviour the ratio of applied impulse to peak force was considered as a possible parameter. The peak force is the maximal force that occurs during impaction and is also referred to later as assembly force. The relationship between the peak force and the applied 
impulse is: the softer the system, the lower the peak force that occurs when the same impulse is applied. Consequently, systems have the same stiffness when the same peak force arises from the same impulse. Based on these considerations, the normalized peak force (NPF), which is the peak force divided by the applied impulse, was introduced as a suitable parameter for the comparison of the damping behaviour. The impulse was calculated by integrating the force function between the points in time when the force exceeds or falls below $5 \%$ of the peak force $\left(t_{5 \%, 1}\right.$ or $\left.t_{5 \%, 2}\right)$. For further explanation the stated parameters are shown in Fig. 5.

\section{Experimental impactions on in vitro model}

A series of trials with 31 surgeons has been carried out with the developed in vitro model. First, each surgeon filled out an anonymous questionnaire providing the following information: sex, handiness, employment in the clinic, number of THA performed per year and the professional experience in years.

Each surgeon was instructed to assemble the head in the same way as they would do under normal intraoperative conditions. The only exception was the cleaning of the taper, because there was no contamination of the taper under laboratory conditions. In each case, the head was placed on the stem, the impactor tool was held against the head and then joined with a single hammer blow. Since these trials were carried out on the in vitro model, the damping behaviour was always the same. Thus, only the peak forces occurring in each case were evaluated as the decisive parameter for the strength of the connection.

Based on the information provided in the questionnaire, the surgeons were divided into different groups. The peak forces achieved in the individual groups were then examined for statistically significant differences using the Students t-test.

\section{Results}

\section{Applied forces during experimental impactions on human cadavers}

The force curves recorded by the two force sensors in hammer and the neck of the stem showed a similar course over all the trials as shown in Fig. 5 as an example. Both courses showed a rapid rise and fall in force. The pulse duration was always below $1 \mathrm{~ms}$. The force measured on the hammer is temporally slightly ahead of the force measured on the neck of the stem. This behaviour can be explained by the inertia of impactor tool and head on the one hand, and by the distance which the head slides onto the taper on the other. When the hammer hits the impactor tool, its inertia and that of the head counteract the inertia of the hammer. The force of the hammer accelerates the impactor tool and the head. As a result the head slides on the taper and a force is built up at the sensor in the neck of the stem. The time offset between the two peaks $\Delta \mathrm{t}_{\mathrm{pp}}$ was $0.29 \mathrm{~ms} \pm 0.14 \mathrm{~ms}$.

Figure 5. Force curves measured by sensors in hammer and neck of the stem while impaction 
Three different surgeons carried out impactions on three different human cadavers. The applied peak forces and impulses are listed in Table 1. None of the experiments resulted in fractures of the femur.

Table 1

Results of impactions on human cadavers

\begin{tabular}{|c|c|c|c|c|c|c|c|c|c|}
\hline \multicolumn{3}{|c|}{ Body Donor } & \multirow[t]{2}{*}{ Side } & \multirow{2}{*}{$\begin{array}{l}\text { Stem } \\
\text { size }\end{array}$} & \multirow[t]{2}{*}{ Surgeon } & \multirow{2}{*}{$\begin{array}{l}F_{\text {peak,hammer }} \\
(\mathrm{N})\end{array}$} & \multirow{2}{*}{$\begin{array}{l}\text { I hammer } \\
\text { (Ns) }\end{array}$} & \multirow{2}{*}{$\begin{array}{l}F_{\text {peak,stem }} \\
(\mathrm{N})\end{array}$} & \multirow{2}{*}{$\begin{array}{l}I_{\text {stem }} \\
\text { (Ns) }\end{array}$} \\
\hline Sex & Age & Weight & & & & & & & \\
\hline \multirow[t]{2}{*}{ female } & 71 & 54 & left & 7 & I & 1733.0 & 0.448 & 1063.6 & 0.367 \\
\hline & & & right & 7 & II & 2881.6 & 0.829 & 2453.4 & 0.679 \\
\hline \multirow[t]{2}{*}{ female } & 83 & 65 & left & 8 & III & 1301.0 & 0.312 & 1127.4 & 0.291 \\
\hline & & & right & 9 & I & 1449.2 & 0.510 & 1402.9 & 0.393 \\
\hline female & 84 & 92 & right & 10 & I & 2122.1 & 0.592 & 1930.0 & 0.495 \\
\hline
\end{tabular}

\section{Normalized peak forces at different mixture ratios}

The in vitro model was built with two different mixing ratios of the gelatine of 1:4 and 1:9 (parts of gelatine:parts of water). Experiments with 10 impactions each were carried out on both models. The NPF of the test series are shown in Fig. 5.

The NPF measured by the hammer showed no significant differences between cadavers and model 1:4 $(p=0.80)$ and between cadavers and model 1:9 $(p=0.56)$.

The NPF measured by the stem are significantly higher on model 1:4 than on the cadavers $\left(p=5.8^{*} 10^{-8}\right)$. In contrast, there are no statistically significant differences between the NPF on the cadavers and on model 1:9 $(p=0.27)$. Therefore, the mixing ratio 1:9 was subsequently used for further experiments with the in vitro model.

Figure 6. Bar chart with determined normalized peak forces

\section{Applied forces during experimental impactions on in vitro model}

Based on the evaluation of the questionnaires completed by the 31 surgeons, the groups shown in Fig. 7 were created. Five female and 26 male surgeons participated in the study. The average professional experience was 7.7 years. Therefore the participants were divided into surgeons with less than eight and equal to or more than eight years of professional experience. The group with less than eight years of professional experience included 17 and the group with 8 and more years included 14 surgeons. Another parameter collected was the number of THAs performed per year. 25 surgeons stated to perform less than 20 THAs per year and only six stated 20 or more. Regarding the handedness no classification was made as only right-handed surgeons participated in the study. 
The comparison of the groups using unpaired T-test showed no significant differences with regard to sex $(p=0.09)$, professional experience $(p=0.71)$ and number of THAs performed per year $(p=0.69)$. The assembly forces of all participants were on average $2037.2 \mathrm{~N} \pm 724.9 \mathrm{~N}$ and ranged from a minimum of $822.5 \mathrm{~N}$ to a maximum of $3835.2 \mathrm{~N}$.

\section{Discussion}

The literature shows that the quality of the head-neck taper junction is influenced by many different factors. Firstly, contamination of the taper by blood or fat decreases the strength of the connection and should therefore be avoided [13]. Furthermore, damage to the taper, e.g. during revision surgery with head replacement, has a negative effect on the junction and can lead to head breakage in metal-ceramic bearings [17]. Therefore an revision head with integrated metal sleeve should always be used in such cases [18]. Another decisive factor is the assembly force. If the assembly force is too low, there is an increased risk of corrosion at the interface due to fretting [19]. Too high assembly forces, on the other hand, can lead to proximal fractures of the femur, which then require extensive surgical treatment [20]. In contrast to the other factors described, there is no clinical standard for eliminating this potential risk. Therefore, the aim of the study was to determine the individual in situ assembly forces of different surgeons and to compare them with the forces recommended in the literature in order to derive a recommendation for action.

In a first step, experimental impactions were carried out on human cadavers. Since the sample size with $\mathrm{N}=5$ trials was very small due to the limited availability of body donors, an in vitro model was developed which reproduced the damping situation of the experiments on the cadavers. This in vitro model was intended to enable a larger number of trials. With the introduced parameter NPF it could be shown that the ratio between the introduced impulse and peak force on the stem was almost identical in the trials on cadavers and on the in vitro model. Therefore it can be assumed that the damping situation was almost identical in both experiments. Reproducing physiological damping behaviour of human soft tissues in vitro not only enables the comparison of the assembly forces of different surgeons, but can also be used to determine the forces actually acting on the patient. For example, Scholl et al [21] have carried out a similar investigation on a very rigid structure and have measured assembly forces up to approximately 25 $\mathrm{kN}$. They explained the very high forces in comparison to other studies [14] by the point where the force was measured (striking pad of the hammer). In this context it should be noted that the participating surgeons considered the striking pad of the hammer used to be too small, which can be seen as a limitation of the experiments. Nevertheless, this study showed that such high forces do not occur neither on the hammer nor on the stem under physiological damping behaviour on the patient. The maximum forces measured on the hammer were $2881.6 \mathrm{~N}$ on cadavers and $4058.3 \mathrm{~N}$ on the model. The measured forces on the hammer were slightly higher than those measured on the stem. This is caused by different bearing situations of impactor tool and stem, friction and also differences between impact direction of the hammer and the neck axis. 
However, the difference accounted for only a few percent. Krull et al [22] also carried out experiments on an in vitro model in the laboratory. They showed that both the stiffness of the tip of impactor tool and the damping of the taper bearing have major influence on the resulting forces. Thus, the damping is a much more significant influencing factor than the point of measurement. In this study, the focus was on the forces measured on the neck of the stem. The force acting on the neck of the stem is the force that counteracts the impact of the hammer and thus the force that is decisive for the Morse taper junction.

As already shown in similar studies, there are considerable differences between the assembly forces of individual surgeons $[16,21]$. The tests on cadavers yielded forces between $1063.9 \mathrm{~N}$ and $2453.4 \mathrm{~N}$. Even larger differences occurred testing on the in vitro model due to the higher number of cases. The forces ranged from a minimum of $822.5 \mathrm{~N}$ to a maximum of $3835.2 \mathrm{~N}$. As the findings show, the range does not depend on sex, age or experience of the surgeons.

Based on the results of other studies, which examined the effects of assembly forces on parameters as pull-out force, turn-off moments and fretting, it appears that the majority of the surgeons involved applied too little force. Based on the resulting pull-out forces from different assembly forces, Ramoutar et al. recommend a minimum assembly force of $2.5 \mathrm{kN}$ [15]. Consequently, $71.0 \%$ of the surgeons would not have applied a sufficiently high assembly force. Based on their findings regarding the relation between assembly force and resulting turn-off moments, Rehmer et al. recommend an assembly force of $4 \mathrm{kN}$ [14]. From their investigations on fretting, Haschke et al. also recommend an assembly force of $4 \mathrm{kN}$ [23]. According to this demand, $100 \%$ of the surgeons showed insufficient assembly forces in this study. However, Rehmer et al. and Haschke et al. also recommend avoiding assembly forces of more than $4 \mathrm{kN}$, as the risk of periprosthetic proximal fractures of the femur increases.

In conclusion, the assembly forces determined show a high variance between surgeons and are low compared to the data given in the literature. As the influences of different head sizes, material combinations or different soft tissue conditions wefre neglected in this study, it can be assumed that the actual variance of the assembly forces is even higher. In our opinion, it is therefore highly recommended to standardize the impaction of the femoral head by means of a new type of surgical instrument.

\section{Conclusions}

The assembly force generated by the surgeon depends on many different factors. One very decisive factor is the damping behaviour of the patient's soft tissue. The tests performed on the in vitro model, which was built based on the actual soft tissue situation, have shown that surgeons apply too little forces to achieve an optimal head-neck taper junction. Because of the inherent risk of periprosthetic femur fractures in case the applied assembly force is too high, surgeons should have an aid, such as a surgical instrument, to ensure that the correct force is always applied. When measuring the assembly forces, we consider it important to measure below the taper, since this is where the force occurs that is decisive for the strength of the Morse taper junction. 


\section{List Of Abbrevations}

\begin{tabular}{|ll|}
\hline THA & Total hip arthroplasty \\
\hline OP & Operation \\
\hline NPF & Normalized peak force \\
\hline DAQ & Data Acquisition \\
PC & Personal Computer \\
PMMA & Polymethylmethacrylate \\
\hline
\end{tabular}

\section{Declarations}

\section{Ethics approval and consent to participate}

Institutional approval was granted for the use the post-mortem tissues in this study under the Saxonian Death and Funeral Act of 1994 (third section, paragraph 18 item 8)

\section{Consent for publication}

Not applicable

\section{Availability of data and materials}

The datasets used and analysed during the current study are available from the corresponding author on reasonable request.

\section{Competing interests}

The authors declare that they have no competing interests.

\section{Funding}

This work was funded by the "Zentrales Innovationsprogramm Mittelstand - ZIM" (Central Innovation Programme for SMEs) no. 944000-033 granted to PD Dr. med. habil. Torsten Prietzel.

\section{Authors' contributions}

TW carried out all experiments and was the major contributor in writing the manuscript. 
TP provided the idea to the study and developed the concept of the study.

RM conducted the statistical analysis of the data.

JPF was a contributor in writing the manuscript.

AR did the literature research.

DZ performed the implantations on cadavers and was a contributor in writing the manuscript.

All authors read and approved the final manuscript.

\section{Acknowledgements}

The authors would like to thank Thomas Wolfskämpf and Matthias Oehme for their support with managing the acquisition of human cadavers for this study. Furthermore, the authors would like to thank the manufacturer Mathys AG for the cooperation and providing the prosthesis components. At least we would like to thank Prof. Stephan Odenwald for his consultation in theoretical issues.

\section{References}

1. Learmonth ID, Young C, Rorabeck C. The operation of the century: Total hip replacement. The Lancet. 2007;370:1508-19. doi:10.1016/S0140-6736(07)60457-7.

2. Statistisches Bundesamt. Die 20 häufigsten Operationen insgesamt. 2018. .

3. Steiner C, Andrews R, Barrett M, Weiss AHCUP, Projections. Mobility/Orthopedic Procedures 2003 to 2012. 2012. http://www.hcup-us.ahrq.gov/reports/projections/2012-03.pdf.

4. Australian Orthopaedic Association. Australian Orthopaedic Association National Joint Replacement Registry. Adelaide; 2012.

5. Gwam CU, Mistry JB, Mohamed NS, Thomas M, Bigart KC, Mont MA, Delanois RE. Current

Epidemiology of Revision Total Hip Arthroplasty in the United States: National Inpatient Sample 2009 to 2013. J Arthroplasty. 2017;32:2088-92. doi:10.1016/j.arth.2017.02.046.

6. Pollock M, Somerville L, Firth A, Lanting B. Outpatient Total Hip Arthroplasty, Total Knee Arthroplasty, and Unicompartmental Knee Arthroplasty: A Systematic Review of the Literature. JBJS Rev. 2016. doi:10.2106/JBJS.RVW.16.00002.

7. Bishop N, Witt F, Pourzal R, Fischer A, Rütschi M, Michel M, Morlock M. Wear patterns of taper connections in retrieved large diameter metal-on-metal bearings. J Orthop Res. 2013;31:1116-22. doi:10.1002/jor.22326.

8. Langton DJ, Sidaginamale R, Lord JK, Nargol AVF, Joyce TJ. Taper junction failure in large-diameter metal-on-metal bearings. Bone Joint Res. 2012;1:56-63. doi:10.1302/2046-3758.14.2000047. 
9. Morlock M, Bünte D, Gührs J, Bishop N. Corrosion of the Head-Stem Taper Junction-Are We on the Verge of an Epidemic?: Review Article. HSS J. 2017;13:42-9. doi:10.1007/s11420-016-9526-4.

10. Kocagöz SB, Underwood RJ, Sivan S, Gilbert JL, Macdonald DW, Day JS, Kurtz SM. Does Taper Angle Clearance Influence Fretting and Corrosion Damage at the Head-Stem Interface? A Matched Cohort Retrieval Study. Semin Arthroplasty. 2013;24:246-54. doi:10.1053/j.sart.2014.01.002.

11. Lakstein D, Kosashvili Y, Backstein D, Safir O, Lee P, Gross AE. Revision total hip arthroplasty with a modular tapered stem. Hip Int. 2010;20:136-42.

12. Meyer H, Mueller T, Goldau G, Chamaon K, Ruetschi M, Lohmann CH. Corrosion at the cone/taper interface leads to failure of large-diameter metal-on-metal total hip arthroplasties. Clin Orthop Relat Res. 2012;470:3101-8. doi:10.1007/s11999-012-2502-5.

13. Lavernia CJ, Baerga L, Barrack RL, Tozakoglou E, Cook SD, Lata L, Rossi MD. The effects of blood and fat on Morse taper disassembly forces. Am J Orthop. 2009;38:187-90.

14. Rehmer A, Bishop NE, Morlock MM. Influence of assembly procedure and material combination on the strength of the taper connection at the head-neck junction of modular hip endoprostheses. Clin Biomech (Bristol Avon). 2012;27:77-83. doi:10.1016/j.clinbiomech.2011.08.002.

15. Ramoutar DN, Crosnier EA, Shivji F, Miles AW, Gill HS. Assessment of Head Displacement and Disassembly Force With Increasing Assembly Load at the Head/Trunnion Junction of a Total Hip Arthroplasty Prosthesis. J Arthroplasty. 2017;32:1675-8. doi:10.1016/j.arth.2016.11.054.

16. Nassutt R, Mollenhauer I, Klingbeil K, Henning O, Grundei H. Relevance of the insertion force for the taper lock reliability of a hip stem and a ceramic femoral head. Biomed Tech (Berl). 2006;51:103-9. doi:10.1515/BMT.2006.018.

17. Pulliam IT, Trousdale RT. Fracture of a ceramic femoral head after a revision operation. A case report. J Bone Joint Surg Am. 1997;79:118-21. doi:10.2106/00004623-199701000-00013.

18. Jennings JM, Dennis DA, Yang CC. Corrosion of the Head-neck Junction After Total Hip Arthroplasty. J Am Acad Orthop Surg. 2016;24:349-56. doi:10.5435/JAAOS-D-15-00111.

19. Panagiotidou A, Cobb T, Meswania J, Skinner J, Hart A, Haddad F, Blunn G. Effect of impact assembly on the interface deformation and fretting corrosion of modular hip tapers: An in vitro study. J Orthop Res. 2018;36:405-16. doi:10.1002/jor.23601.

20. Sakai R, Takahashi A, Takahira N, Uchiyama K, Yamamoto T, Uchida K, et al. Hammering force during cementless total hip arthroplasty and risk of microfracture. Hip Int. 2011;21:330-5. doi:10.5301/HIP.2011.8408.

21. Scholl L, Schmidig G, Faizan A, TenHuisen K, Nevelos J. Evaluation of surgical impaction technique and how it affects locking strength of the head-stem taper junction. Proc Inst Mech Eng $\mathrm{H}$. 2016;230:661-7. doi:10.1177/0954411916644477.

22. Krull A, Morlock MM, Bishop NE. Maximizing the fixation strength of modular components by impaction without tissue damage. Bone Joint Res. 2018;7:196-204. doi:10.1302/2046-3758.72.BJR2017-0078.R2. 
23. Haschke H, Jauch-Matt SY, Sellenschloh K, Huber G, Morlock MM. Assembly force and taper angle difference influence the relative motion at the stem-neck interface of bi-modular hip prostheses. Proc Inst Mech Eng H. 2016;230:690-9. doi:10.1177/0954411916648717.

\section{Figures}

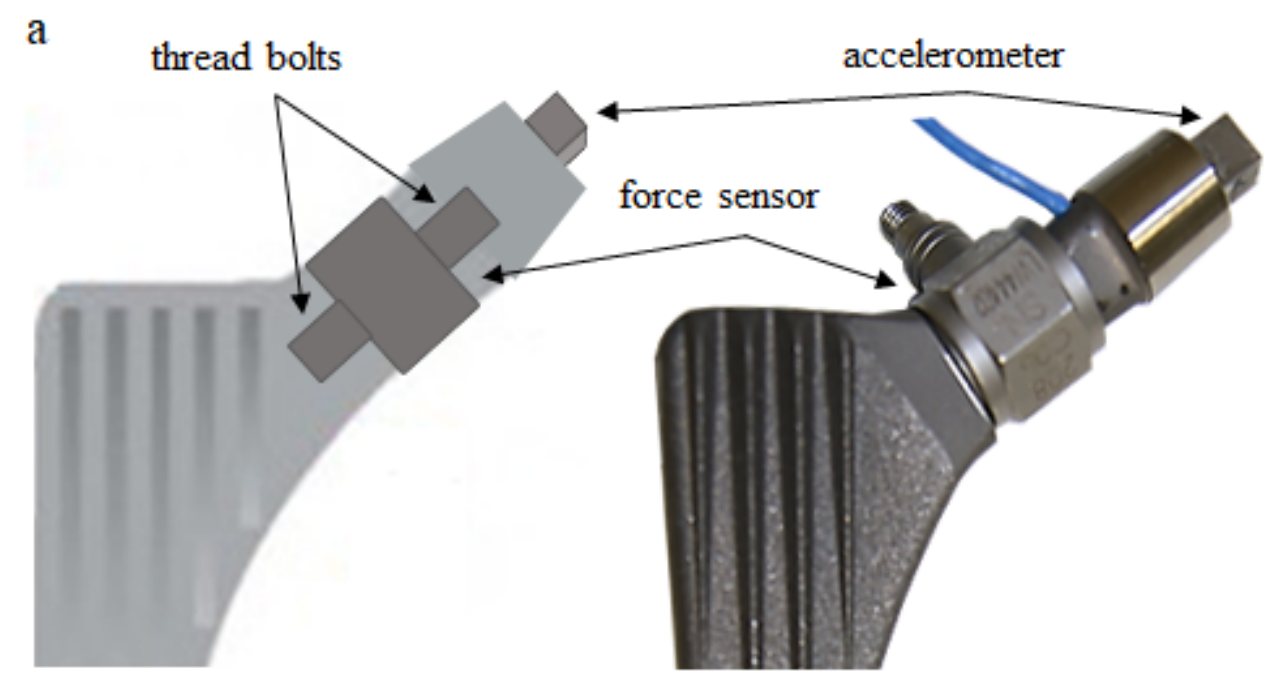

$\mathrm{b}$

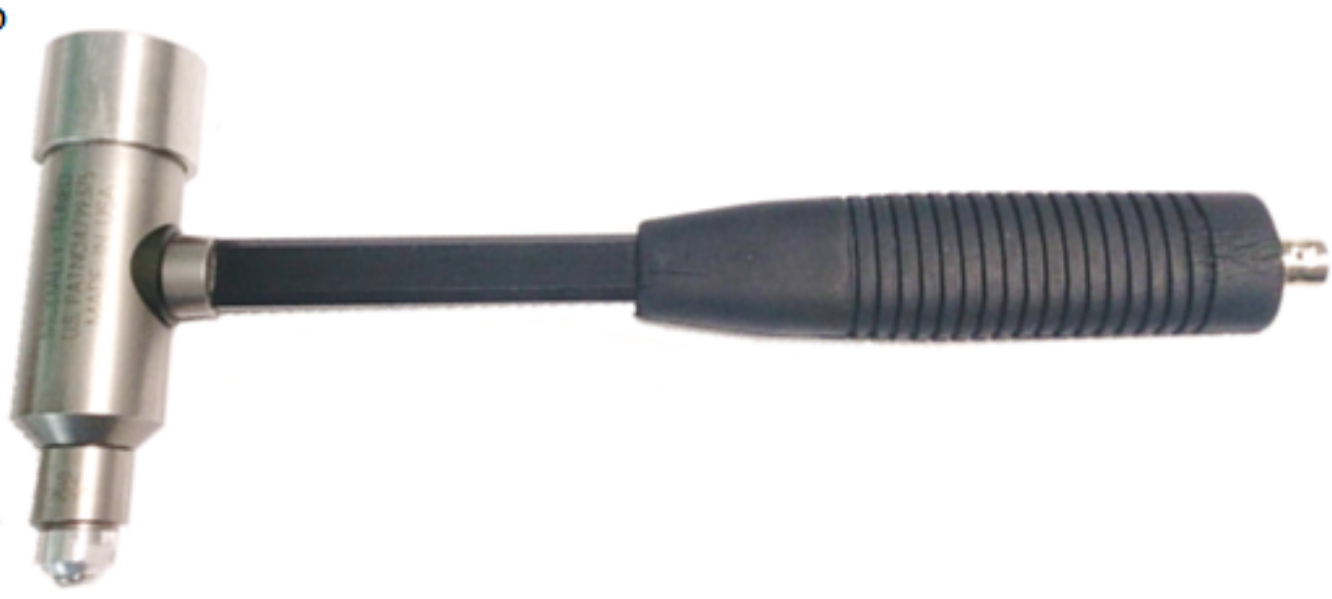

Figure 1

a) Stem with integrated piezoelectric force sensor; b) Impulse hammer 

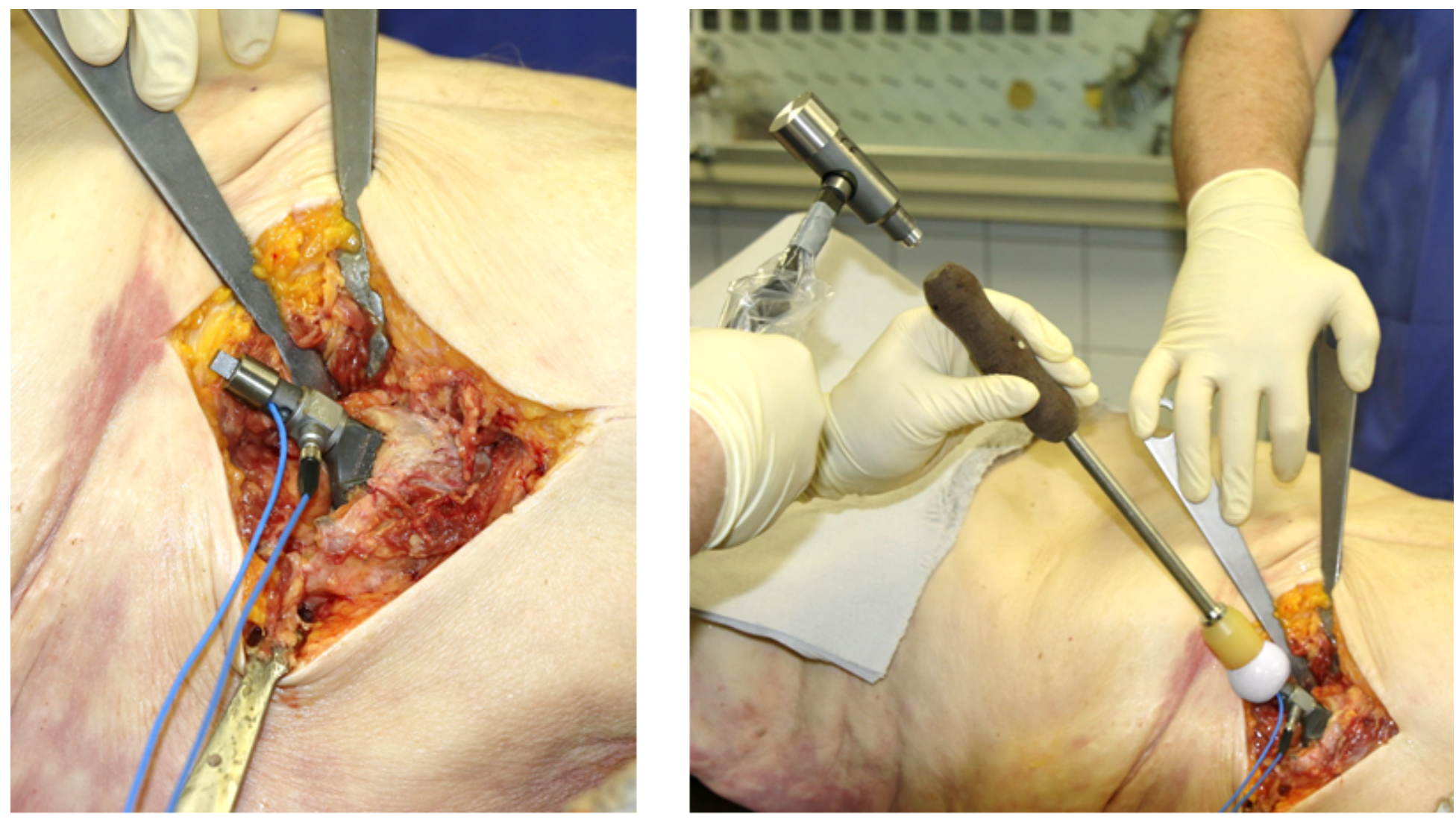

Figure 2

left: Implanted measuring stem; right: Setup to measure in situ assembly forces

Gelatine in

PMMA housing

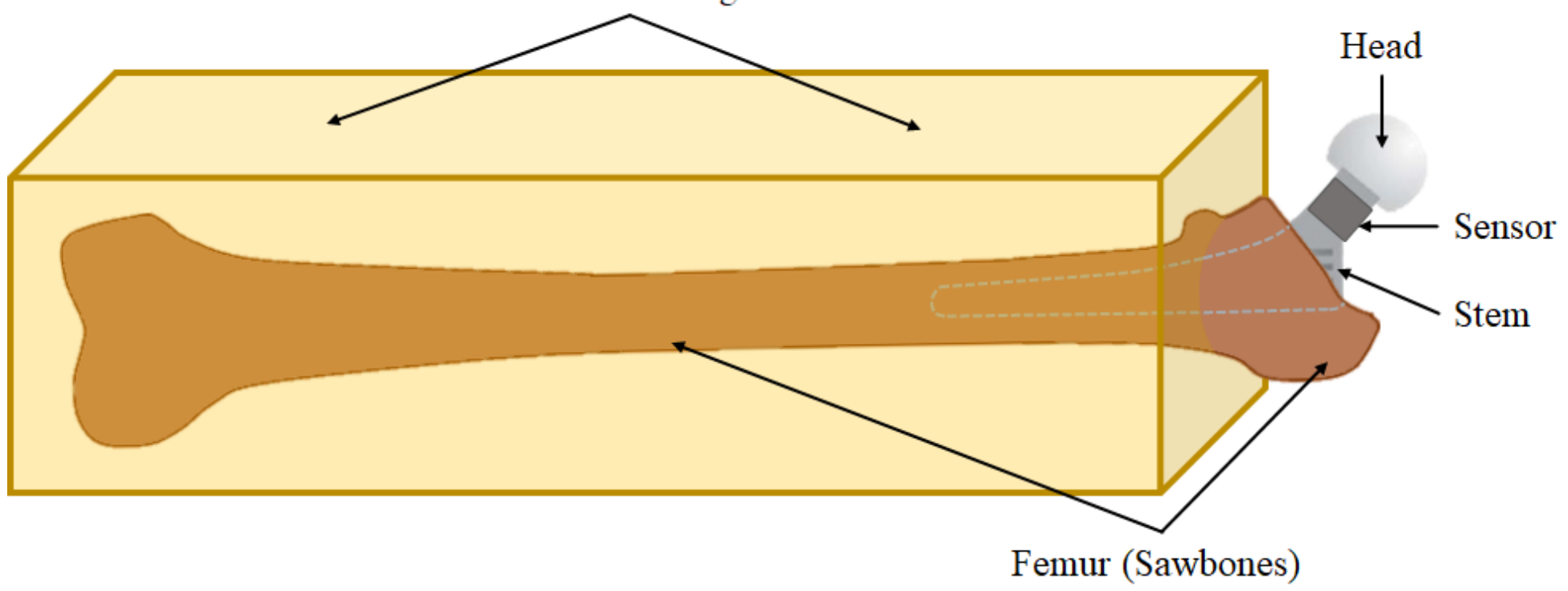

Figure 3

Schematic of embedded Femur with implanted THA stem 


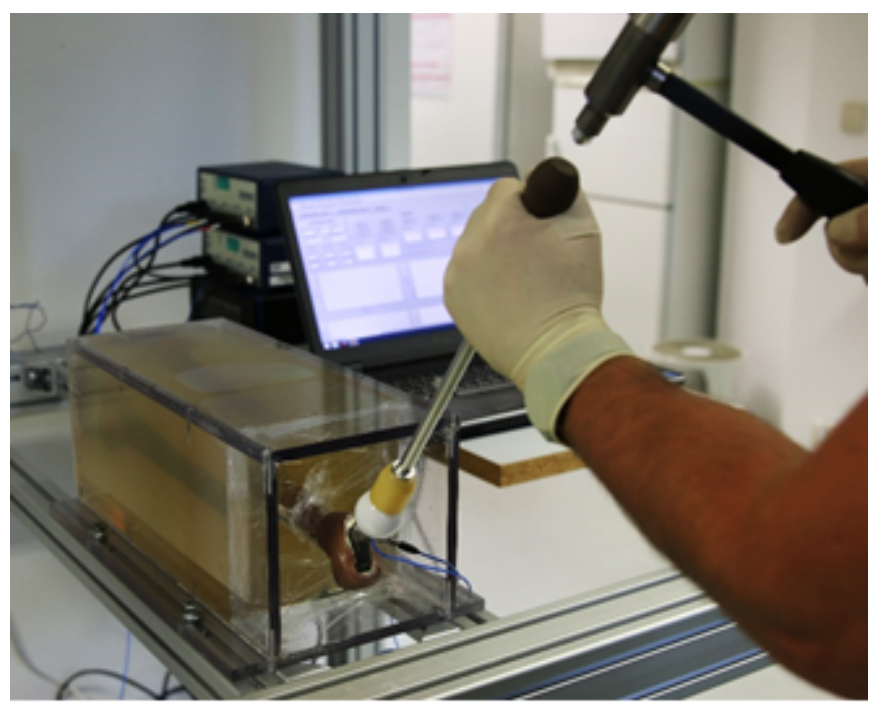

Figure 4

Final in vitro model

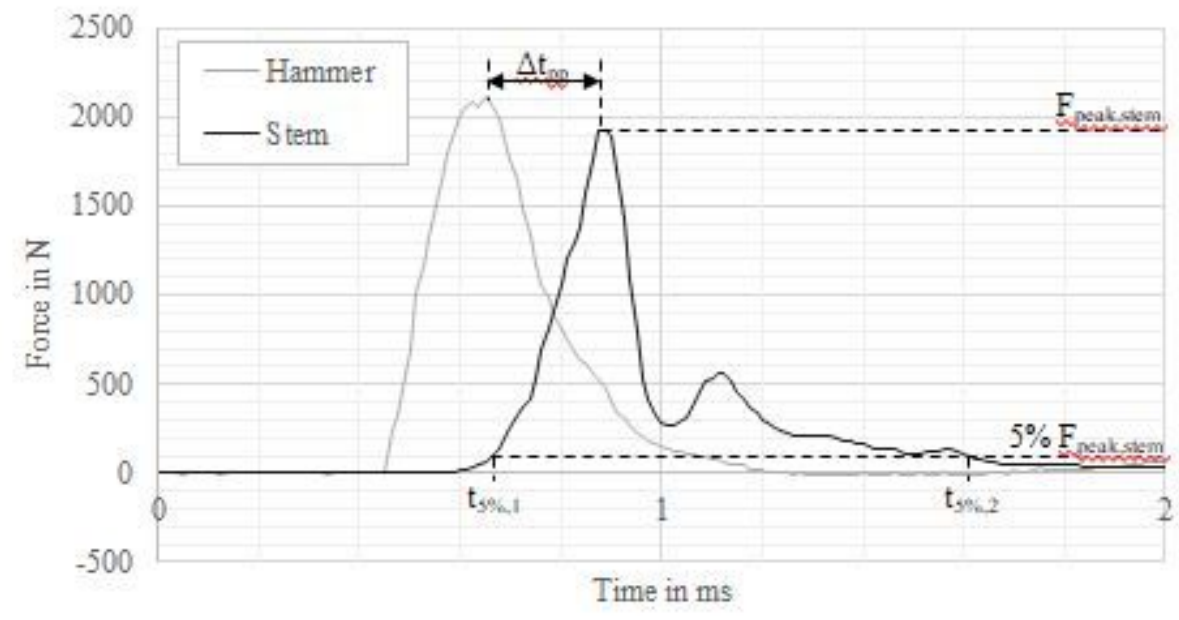

Figure 5

Force curves measured by sensors in hammer and neck of the stem while impaction 


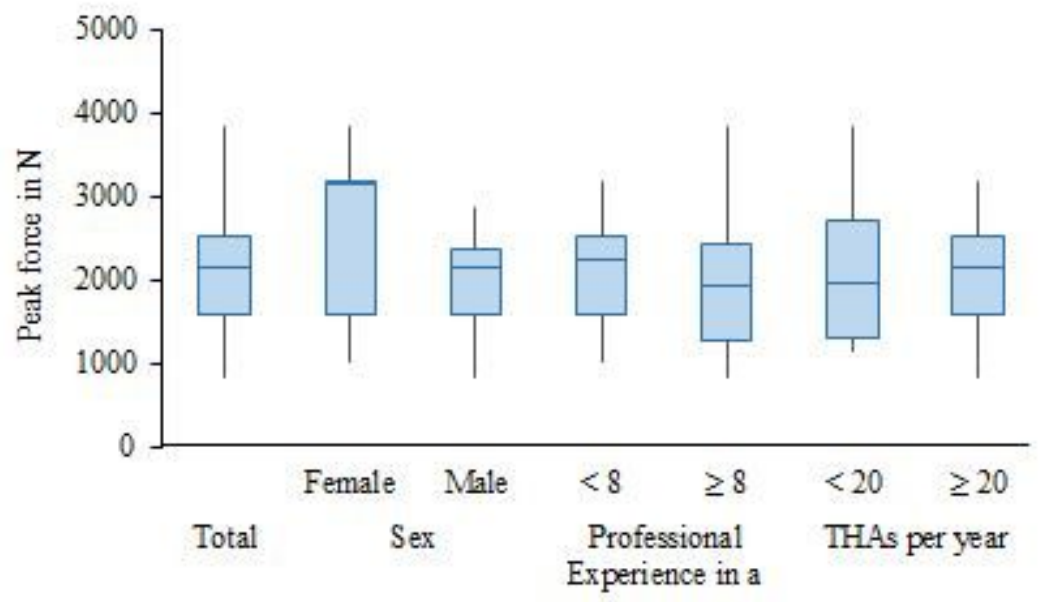

Figure 6

Bar chart with determined normalized peak forces

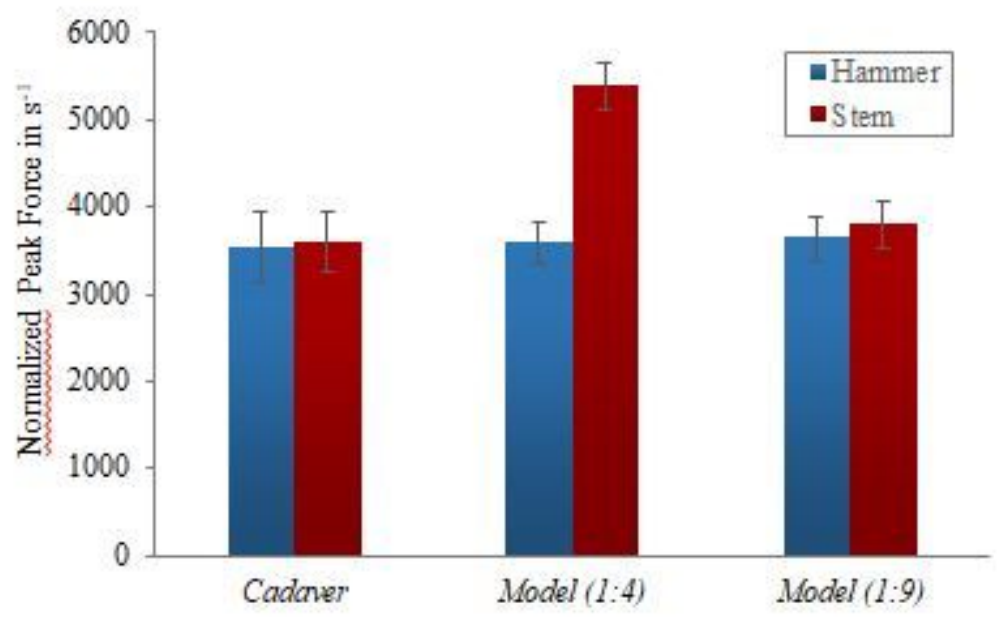

Figure 7

Bar chart with determined peak forces in several groups of surgeons 